\title{
Segmental and Chain Dynamics of Isotactic Polypropylene Melts
}

Georgia Evangelia Logotheti and Doros N. Theodorou

\section{Supporting Information}

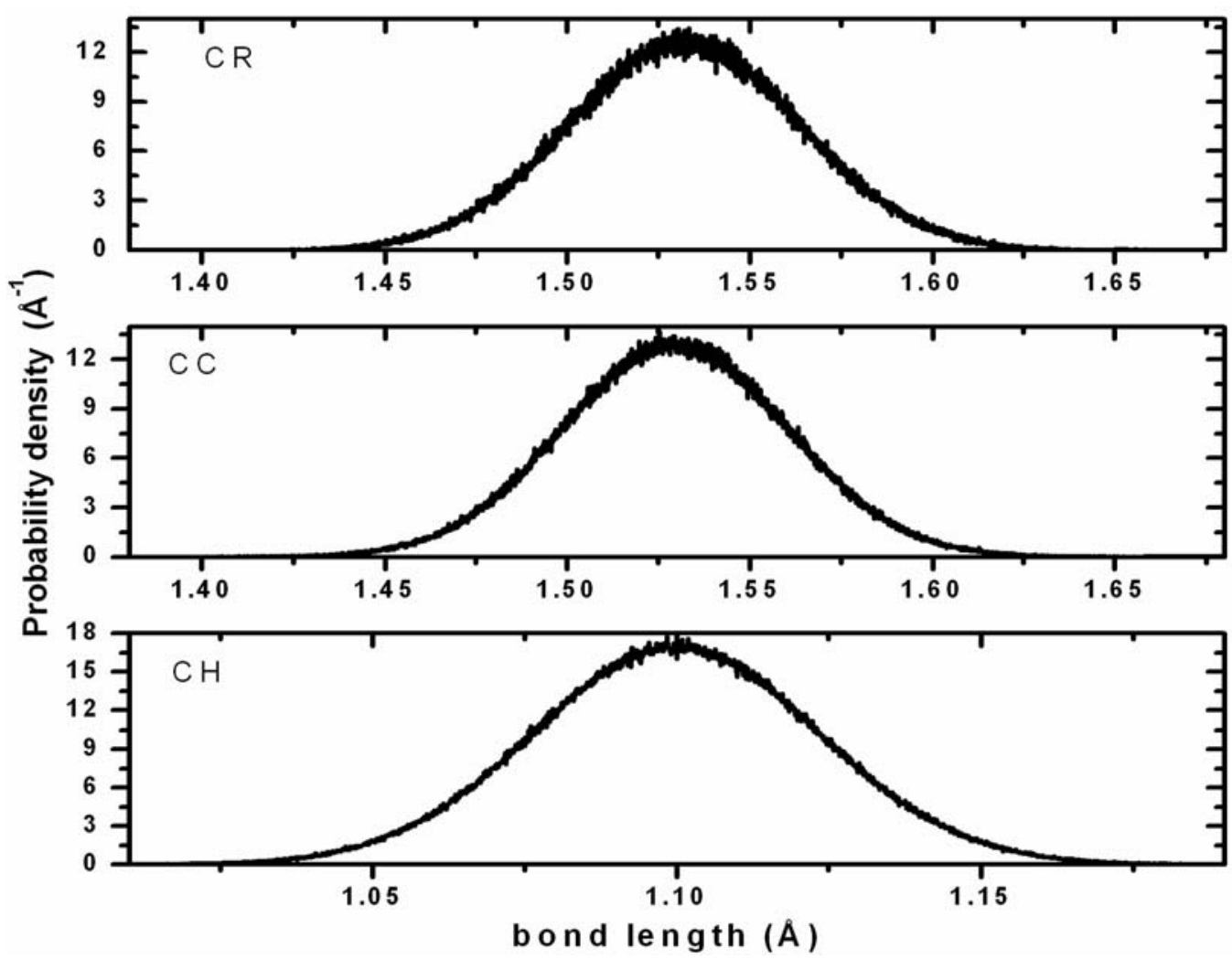

Figure S.M. 1: Simulated bond length distributions in iPP melt. Results were obtained from NPT simulations at $400 \mathrm{~K}$ and atmospheric pressure. 

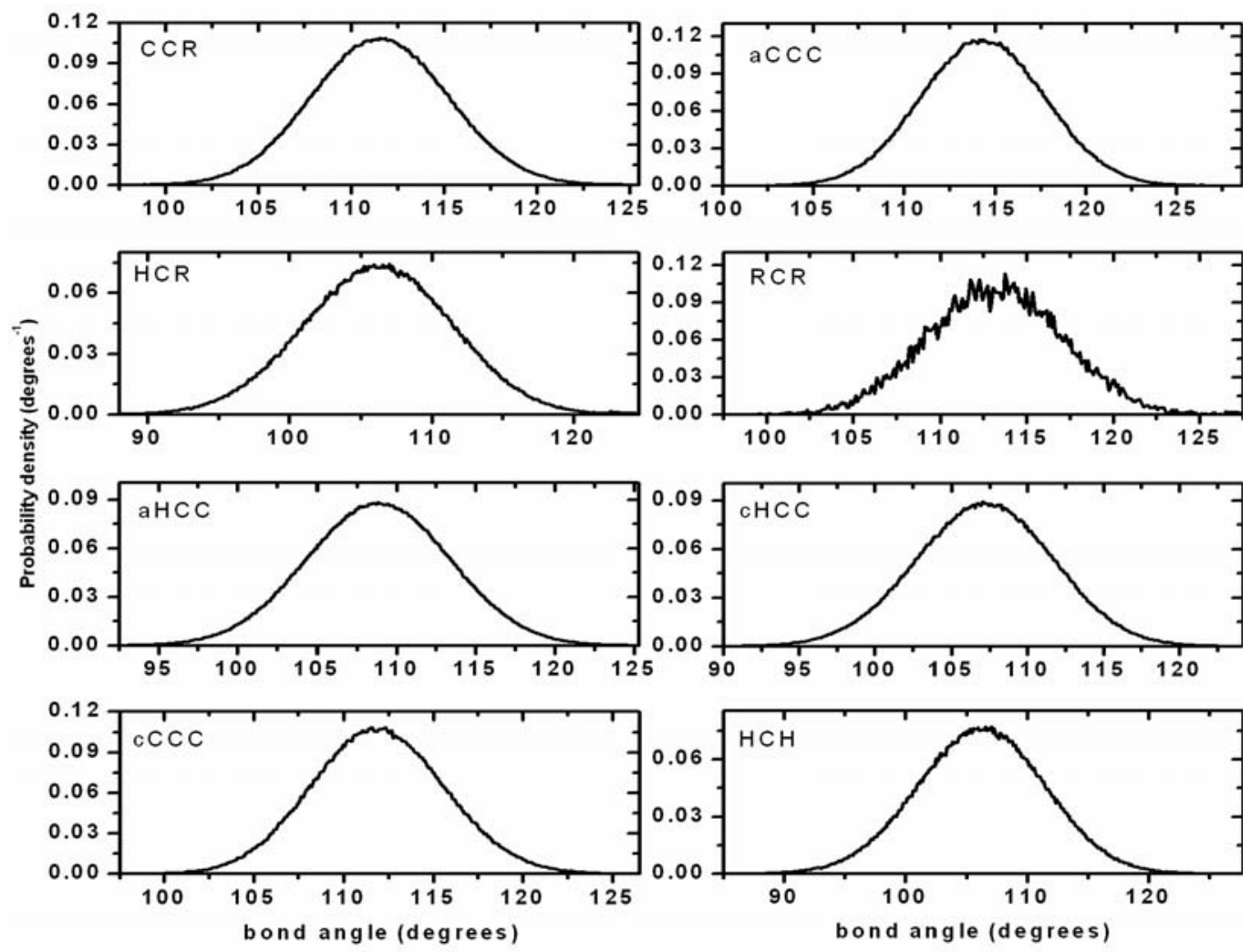

Figure S.M. 2: Simulated bond angle distributions in iPP melt. Results were obtained from NPT simulations at $400 \mathrm{~K}$ and atmospheric pressure. 

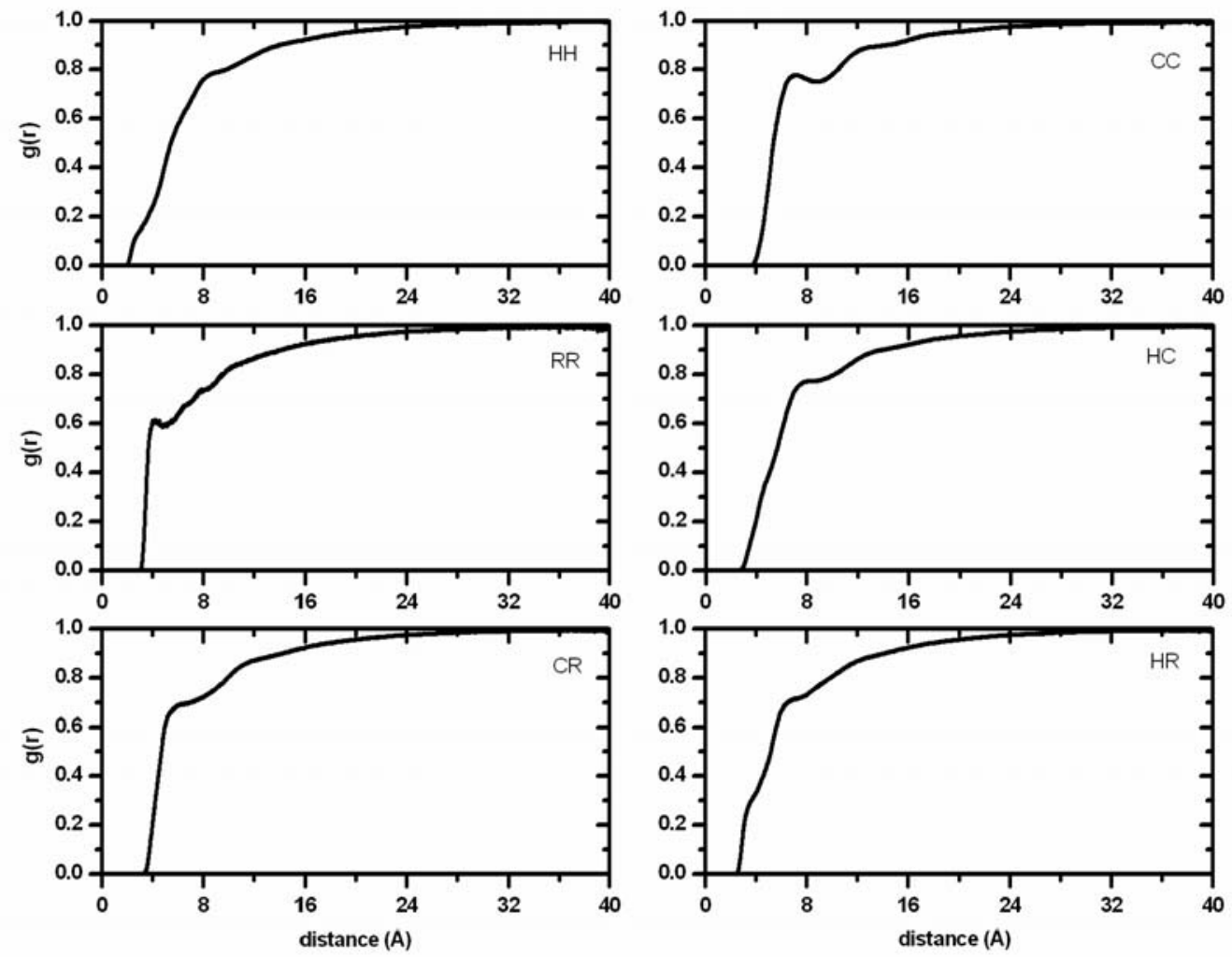

Figure S.M. 3: Intermolecular pair distribution functions $g(r)$ at $T=450 \mathrm{~K}$ and $P=1$ atm. Results were obtained from NPT simulations conducted using the 16 molecule model system. 


\section{Conformational Probabilities for pairs of adjacent bonds}

The á priori conformational probabilities for the bond pair $(i-1, i)$ were calculated using the five state RIS model for isotactic polypropylene with the parameters proposed by Suter and Flory, ${ }^{30,31}$ for both dyad types. The same probabilities were also extracted by analyzing the simulation runs. Results for each type of dyad at $T=450 \mathrm{~K}$ and atmospheric pressure are listed in Table S.M. I. The same calculations were repeated using a simpler, three-state RIS model ${ }^{32-35}$ for isotactic polypropylene with the parameters proposed from Biskup and Cantow, ref 34. In the simulation, assignments of torsion angles to the 3 discrete torsional states $(\mathrm{t}, \mathrm{g}, \bar{g})$ were made according to ref 34 . Results are given in Table S.M. II.

Table S.M. I: Á priori probabilities of pairs of rotational states adopted by dyads, as calculated using the five state RIS model and as extracted from simulation trajectories at $450 \mathrm{~K}$ and $1 \mathrm{~atm}$.

\begin{tabular}{|c|c|c|c|c|c|c|c|c|c|c|}
\hline \multicolumn{11}{|c|}{ Dyad type: -( $\left.\mathrm{CH}_{2}-\mathrm{CHR}-\mathrm{CH}_{2}-\mathrm{CHR}-\mathrm{CH}_{2}\right)-$} \\
\hline \multirow{5}{*}{$\begin{array}{l}\bar{\Xi} \\
\mathscr{O} \\
\Xi \\
\mathscr{\Omega} \\
\widetilde{\Omega}\end{array}$} & $t t$ & 0.000 & $t^{*} t$ & 0.119 & $g^{*} t$ & 0.000 & $g t$ & 0.320 & $\bar{g} t$ & 0.000 \\
\hline & $t t^{*}$ & 0.119 & $t^{*} t^{*}$ & 0.000 & $g^{*} t^{*}$ & 0.000 & $g t^{*}$ & 0.000 & $\bar{g} t^{*}$ & 0.028 \\
\hline & $\operatorname{tg}^{*}$ & 0.000 & $t^{*} g^{*}$ & 0.000 & $g^{*} g^{*}$ & 0.000 & $g g^{*}$ & 0.021 & $\bar{g} g^{*}$ & 0.012 \\
\hline & $\operatorname{tg}$ & 0.320 & $t^{*} g$ & 0.000 & $g^{*} g$ & 0.021 & $g g$ & 0.000 & $\bar{g} g$ & 0.000 \\
\hline & $t \bar{g}$ & 0.000 & $t^{*} \bar{g}$ & 0.028 & $g^{*} \bar{g}$ & 0.012 & $g \bar{g}$ & 0.000 & $\bar{g} \bar{g}$ & 0.000 \\
\hline \multirow{5}{*}{ 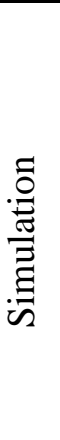 } & $t t$ & 0.117 & $t^{*} t$ & 0.069 & $g^{*} t$ & 0.042 & $g t$ & 0.265 & $\bar{g} t$ & 0.017 \\
\hline & $t t^{*}$ & 0.069 & $t^{*} t^{*}$ & 0.003 & $g^{*} t^{*}$ & 0.003 & $g t^{*}$ & 0.019 & $\bar{g} t^{*}$ & 0.016 \\
\hline & $\operatorname{tg}^{*}$ & 0.042 & $t^{*} g^{*}$ & 0.003 & $g^{*} g^{*}$ & 0.000 & $g g^{*}$ & 0.003 & $\bar{g}{ }^{*}$ & 0.004 \\
\hline & $\operatorname{tg}$ & 0.265 & $t^{*} g$ & 0.019 & $g^{*} g$ & 0.003 & $g g$ & 0.001 & $\bar{g} g$ & 0.006 \\
\hline & $t \bar{g}$ & 0.017 & $t^{*} \bar{g}$ & 0.016 & $g^{*} \bar{g}$ & 0.004 & $g \bar{g}$ & 0.006 & $\bar{g} \bar{g}$ & 0.000 \\
\hline
\end{tabular}




\begin{tabular}{|c|c|c|c|c|c|c|c|c|c|c|}
\hline \multicolumn{11}{|c|}{ Dyad type: -(CHR - $\left.\mathrm{CH}_{2}-\mathrm{CHR}-\mathrm{CH}_{2}-\mathrm{CHR}\right)-$} \\
\hline \multirow{5}{*}{ 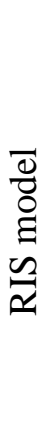 } & $t t$ & 0.124 & $t^{*} t$ & 0.042 & $g^{*} t$ & 0.023 & $g t$ & 0.238 & $\bar{g} t$ & 0.012 \\
\hline & $t t^{*}$ & 0.042 & $t^{*} t^{*}$ & 0.014 & $g^{*} t^{*}$ & 0.008 & $g t^{*}$ & 0.080 & $\bar{g} t^{*}$ & 0.004 \\
\hline & $\operatorname{tg}^{*}$ & 0.023 & $t^{*} g^{*}$ & 0.008 & $g^{*} g^{*}$ & 0.000 & $g g^{*}$ & 0.000 & $\bar{g} g^{*}$ & 0.002 \\
\hline & $\operatorname{tg}$ & 0.238 & $t^{*} g$ & 0.080 & $g^{*} g$ & 0.000 & $g g$ & 0.000 & $\bar{g} g$ & 0.022 \\
\hline & $t \bar{g}$ & 0.012 & $t^{*} \bar{g}$ & 0.004 & $g^{*} \bar{g}$ & 0.002 & $g \bar{g}$ & 0.022 & $\bar{g} \bar{g}$ & 0.000 \\
\hline \multirow{5}{*}{ 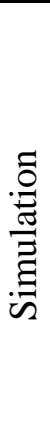 } & $t t$ & 0.218 & $t^{*} t$ & 0.040 & $g^{*} t$ & 0.028 & $g t$ & 0.210 & $\bar{g} t$ & 0.016 \\
\hline & $t t^{*}$ & 0.040 & $t^{*} t^{*}$ & 0.004 & $g^{*} t^{*}$ & 0.005 & $g t^{*}$ & 0.050 & $\bar{g} t^{*}$ & 0.007 \\
\hline & $\operatorname{tg}^{*}$ & 0.028 & $t^{*} g^{*}$ & 0.005 & $g^{*} g^{*}$ & 0.001 & $g g^{*}$ & 0.017 & $\bar{g} g^{*}$ & 0.001 \\
\hline & $\operatorname{tg}$ & 0.210 & $t^{*} g$ & 0.050 & $g^{*} g$ & 0.017 & $g g$ & 0.001 & $\bar{g} g$ & 0.014 \\
\hline & $t \bar{g}$ & 0.016 & $t^{*} \bar{g}$ & 0.007 & $g^{*} \bar{g}$ & 0.001 & $g \bar{g}$ & 0.014 & $\bar{g} \bar{g}$ & 0.000 \\
\hline
\end{tabular}

Table S.M. II: Á priori probabilities of pairs of rotational states adopted by dyads, as calculated using the three-state RIS model and as extracted from simulation trajectories at $450 \mathrm{~K}$ and $1 \mathrm{~atm}$.

\begin{tabular}{|c|c|c|c|c|c|c|c|c|c|c|c|c|}
\hline \multicolumn{7}{|c|}{ Dyad type: -( $\left.\mathrm{CH}_{2}-\mathrm{CHR}-\mathrm{CH}_{2}-\mathrm{CHR}-\mathrm{CH}_{2}\right)-$} & \multicolumn{6}{|c|}{ Dyad type: -(CHR - $\left.\mathrm{CH}_{2}-\mathrm{CHR}-\mathrm{CH}_{2}-\mathrm{CHR}\right)-$} \\
\hline$\sqrt{-1}$ & $t t$ & 0.051 & $g t$ & 0.406 & $\bar{g} t$ & 0.044 & $t t$ & 0.152 & $g t$ & 0.330 & $\bar{g} t$ & 0.019 \\
\hline 吕 & $\operatorname{tg}$ & 0.406 & $g g$ & 0.017 & $\bar{g} g$ & 0.015 & $\operatorname{tg}$ & 0.330 & $g g$ & 0.066 & $\bar{g} g$ & 0.042 \\
\hline 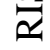 & $t \bar{g}$ & 0.044 & $g \bar{g}$ & 0.015 & $\bar{g} \bar{g}$ & 0.002 & $t \bar{g}$ & 0.019 & $g \bar{g}$ & 0.042 & $\bar{g} \bar{g}$ & 0.000 \\
\hline$=$ & $t t$ & 0.263 & $g t$ & 0.326 & $\bar{g} t$ & 0.029 & $t t$ & 0.303 & $g t$ & 0.293 & $\bar{g} t$ & 0.024 \\
\hline$\frac{\pi}{3}$ & $\operatorname{tg}$ & 0.326 & $g g$ & 0.008 & $\bar{g} g$ & 0.009 & tg & 0.293 & $g g$ & 0.035 & $\bar{g} g$ & 0.014 \\
\hline "ज & $t \bar{g}$ & 0.029 & $g \bar{g}$ & 0.009 & $\bar{g} \bar{g}$ & 0.001 & $t \bar{g}$ & 0.024 & $g \bar{g}$ & 0.014 & $\bar{g} \bar{g}$ & 0.000 \\
\hline
\end{tabular}

